\title{
ON A GENERAL ARITHMETIC FORMULA OF LIOUVILLE.
}

BY PROFESSOR E. T. BELL.

(Read before the American Mathematical Society April 9, 1921.)

1. Introduction. In his History of the Theory of Numbers (vol. 2, chap. 11), Dickson gives a resumé of the present state of the celebrated general formulas of Liouville in the theory of numbers, and remarks that the only formula for which no proof has been published is $(Q)$ of the sixth article. Some years ago I developed an analytic method of complete generality suitable for dealing with all such arithmetic questions, and I applied it incidentally to the proofs of Liouville's formulas. By means of this method we may immediately paraphrase any identity between elliptic, abelian or theta functions, provided only that it contains the arguments of the functions and is not merely an identity between constants, into another identity between arithmetic functions of the greatest generality, such, for example, as those considered by Liouville.

A detailed account of the general principles upon which the method is given in my paper in the January number of the Transactions. In a continuation of the same paper which is to appear later, there is a selection of illustrative examples including some of Liouville's formulas and others of new kinds. Since the formula $(Q)$ is not among these, however, I shall give a proof of it here. This formula is in fact unique among all of Liouville's, for it is the only one which depends immediately upon the addition theorems for the elliptic functions. It will be necessary first to recall a very special case of a general theorem established in the paper just mentioned.

2. A general Theorem. Let $f(x, y)$ denote a function of the arguments $x, y$ which takes a single definite value whenever $x$ and $y$ are positive, zero or negative integers, and let the $a, b, c$, and $d$ denote integers. Then if the following equation is an identity in $u$ and $v, \Sigma_{i} a_{i} \sin b_{i} u \sin c_{i} v=0$, we can infer that $\Sigma_{i} a_{i} f\left(b_{i}, c_{i}\right)=0$, for $f(x, y)$ defined as above and subjected to the conditions

$f(x, y)=-f(-x, y)=-f(x,-y), \quad f(0, y)=0=f(x, 0)$.

Beyond these conditions $f(x, y)$ is general in the widest sense. A proof of this result will be found in the paper cited above. 
3. The Formula $(Q)$ of Liouville. Let us write for brevity $\mu=2 K / \pi, k \mu=h$, and consider the following obvious identity, in which accents signify derivatives with respect to $u$ or with respect to $v$,

$$
\begin{array}{r}
h \operatorname{sn} \mu(u+v)\left[(h \operatorname{sn} \mu u)(h \operatorname{sn} \mu v)^{\prime}-(h \operatorname{sn} \mu v)(h \operatorname{sn} \mu u)^{\prime}\right] \\
=h \operatorname{sn} \mu(u-v)\left[(h \operatorname{sn} \mu u)(h \operatorname{sn} \mu v)^{\prime}+(h \operatorname{sn} \mu v)(h \operatorname{sn} \mu u)^{\prime}\right] .
\end{array}
$$

From the classical series for sn $\mu u$ we have at once

$h \operatorname{sn} \mu u=4 \Sigma q^{m / 2}(\Sigma \sin d u), \quad(h \operatorname{sn} \mu u)^{\prime}=4 \Sigma q^{m / 2}(\Sigma d \cos d u)$, where the first summation extends to all $m=1,3,5, \cdots$, and the second to all the divisors $1, d, \cdots, m$ of $m$. Replacing in the identity the $s n, s n^{\prime}$ functions by their equivalent series and then equating coefficients of like powers of $q$, we find after some easy reductions the following identity in $u, v$,

$\Sigma d_{3}\left[\sin \left(d_{1}+d_{3}\right) u \sin \left(d_{1}+d_{2}\right) v-\sin \left(d_{1}+d_{2}\right) u \sin \left(d_{1}+d_{3}\right) v\right.$

$\left.+\sin \left(s_{1}-d_{3}\right) u \sin \left(d_{1}+d_{2}\right) v-\sin \left(d_{1}+d_{2}\right) u \sin \left(d_{1}-d_{3}\right) v\right]$ $=\Sigma d_{3}\left[\sin \left(d_{1}+d_{3}\right) u \sin \left(d_{1}-d_{2}\right) v-\sin \left(d_{1}-d_{2}\right) u \sin \left(d_{1}+d_{3}\right) v\right.$ $\left.+\sin \left(d_{1}-d_{3}\right) u \sin \left(d_{1}-d_{2}\right) v-\sin \left(d_{1}-d_{2}\right) u \sin \left(d_{1}-d_{3}\right) v\right]$,

where the summations refer to all positive divisors $d_{1}, d_{2}, d_{3}$ defined by

$m=m_{1}+m_{2}+m_{3}, \quad m_{1}=d_{1} \delta_{1}, \quad m_{2}=d_{2} \delta_{2}, \quad m_{3}=d_{3} \delta_{3}$, $m, m_{1}, m_{2}, m_{3}$ being odd and positive and $m$ constant. Hence by the theorem quoted in $\S 2$,

$$
\begin{aligned}
& \Sigma d_{3}\left[f \left(d_{1}+d_{3}, d_{1}\right.\right.\left.+d_{2}\right)-f\left(d_{1}+d_{2}, d_{1}+d_{3}\right) \\
&\left.+f\left(d_{1}-d_{3}, d_{1}+d_{2}\right)-f\left(d_{1}+d_{2}, d_{1}-d_{3}\right)\right] \\
&=\Sigma d_{3}\left[f\left(d_{1}+d_{3}, d_{1}-d_{2}\right)-f\left(d_{1}-d_{2}, d_{1}+d_{3}\right)\right. \\
&+
\end{aligned}
$$

Now putting $\psi(x, y)=f(x, y)-f(y, x)$, we see that $\psi(x, y)$ has a single determinate value whenever $x$ and $y$ are positive, zero or negative integers, and that $\psi(x, y)$ satisfies the conditions

$$
\begin{gathered}
\psi(-x, y)=-\psi(x, y)=\psi(x,-y)=\psi(y, x), \\
\psi(0, y)=0=\psi(x, 0) .
\end{gathered}
$$


Moreover, if $\psi(x, y)$ is the most general function satisfying all of these conditions, we can put $\psi(x, y)=f(x, y)-f(y, x)$ without loss of generality. A brief discussion of such questions is given in section III of the paper cited. Hence from the identity for $f(x, y)$, we infer at once that

$$
\begin{aligned}
& \Sigma d_{3}\left[\psi\left(d_{1}+d_{3}, d_{1}+d_{2}\right)+\psi\left(d_{1}-d_{3}, d_{1}+d_{2}\right)\right] \\
& =\Sigma d_{3}\left[\psi\left(d_{1}+d_{3}, d_{1}-d_{2}\right)+\psi\left(d_{1}-d_{3}, d_{1}-d_{2}\right)\right],
\end{aligned}
$$

and this is the formula $(Q)$ of Liouville.

The 39 forms of the addition theorems given by Jacobi in section 18 of the Fundamenta Nova imply a multitude of such consequences, many of which are of arithmetic interest.

The author wishes to express his indebtedness to Professor Frank Nelson Cole for encouragement and inspiration, not only in this paper, but for much of his other mathematical work.

UNIVERSITY OF WASHINGTON, January 19, 1921.

\section{SHORTER NOTICES}

General Theory of Polyconic Projections. By Oscar S. Adams. Washington, United States Coast and Geodetic Survey, 1919. Special Publication No. 57. 174 pp.

There are many ways of representing, or projecting, the surface of the earth, or parts of it, upon a plane. Any system of lines may be chosen to represent the parallels of latitude, and a second system to represent the meridians. The book before us is designed to give a full account of the so-called polyconic projection, that is the projection in which parallels of latitude are represented by arcs of a non-concentric system of circles with collinear centers. The line of centers is usually, but not necessarily, taken for the central, or principal, meridian. The mathematical problem consists in setting up the equations for the meridians under various hypotheses, methods for constructing the meridians, spacing the parallels, determining the magnification, and so forth. These details the author has worked out for various cases, deriving the formulas for the ellipsoid as well as for the sphere.

Stereographic projection is one type of polyconic projection. 